\title{
Adrenal Pseudocyst: Case Report
}

\author{
Frais Alaswad* \\ Specialist General Surgeon, NMC Speciality Hospital, UAE
}

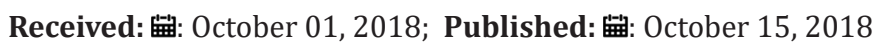

*Corresponding author: Frais Alaswad, FRCS(ENG), FRCSI(IRL), FEBS(EU), FACS, NMC Speciality Hospital, Dubai, UAE

\begin{abstract}
Pseudocyst type of adrenal cysts is most common; however, this type of ectopic cyst is quite rare in excessively large adrenal cysts. A 35-yearold woman was admitted to our institution with distension of her right flank and the upper quadrant of her abdomen. Abdominal ultrasonography revealed a cystic lesion with a homogenous anechoic texture, and measuring $28 \mathrm{~cm}$ in diameter. Computed tomography imaging displayed a giant cystic lesion adjacent to the liver, pancreas, kidney. The origin of the cyst was not identified. We were not able to make a preoperative diagnosis; therefore, the patient underwent resection of the mass by open laparotomy for therapeutic diagnosis. Intraoperatively, the mass was identified to be cystic and adhered to the right adrenal gland. It was filled with more than $2500 \mathrm{~mL}$ of serous brown fluid. The content of the cyst contained no atypical cells on cytological examination, and the cyst was pathologically classified as a pseudocyst cyst. We describe a case of a patient with a giant pseudocyst of the adrenal gland. The present data suggest that surgeons should decide treatment strategy for large adrenal cysts in consideration of hormonal function, degree of size, and possibility of malignancy.
\end{abstract}

\section{Introduction}

The first case of an adrenal cyst was reported in 1670 by Greaseless whose patient presented with abdominal pain and a palpable mass [1]. Adrenal cysts are generally rare, and around 600 cases have been reported in the literature so far [2] and [3]. The incidence of adrenal cysts in autopsy series ranges from $0.06 \%$ to $0.18 \%$. They are usually non-functional, asymptomatic and less than $10 \mathrm{~cm}$ in diameter when discovered incidentally. However, giant adrenal cysts are cysts of the adrenal gland which are larger than $10 \mathrm{~cm}$ in diameter [4] and [5]. They vary greatly in size and may be as large as $50 \mathrm{~cm}$ in diameter and contain many litres of fluid. There is no side predilection for the right or left adrenal gland. Giant adrenal cysts pose a diagnostic conundrum to the surgeon as localization of their origin is very difficult [4-6]. They may occur at any age, but most of them are seen in the 3rd to 4th decades of life with a higher preponderance in females [2-5,7], just like in our patient. In some series, a female preponderance of about 3:1 was noted for unknown reasons [7].

Traditionally, adrenal cysts are divided into neoplastic and nonneoplastic groups. Non-neoplastic adrenal cysts may be further categorized as any of the 4 major types: pseudocyst (39\%), epithelial (9\%), parasitic (7\% generally echinococcal), or endothelial cysts $(45 \%)[4,8,9]$. The most common types are endothelial cysts and adrenal pseudocysts which represent the encapsulated remains of adrenal hemorrhage. Amongst the different types of adrenal cystic lesions, adrenal pseudocysts are the most common. Adrenal pseudocysts are cystic lesions which arise within the adrenal gland.
They are surrounded by a fibrous wall devoid of a recognizable lining layer. They are characterized by the absence of an epithelial lining $[2,9,12]$. Most endothelial cysts, on the other hand, are either of lymphatic or vascular origin and result from developmental malformations, namely hemangiomas or lymphangiomas [7]. The etiology of adrenal pseudocysts is not known, however several theories have been put forward such as cystic degeneration of a primary adrenal neoplasm, vascular neoplasm and malformation as well as hemorrhage of adrenal veins into the adrenal gland $[2,9,12]$.

Parasitic adrenal cysts, on the other hand, are principally echinococcal in origin and found in settlements endemic for hydatid disease [13]. In our patient we could not ascertain the possible etiology of the adrenal pseudocyst. Most adrenal cysts are asymptomatic and less than $10 \mathrm{~cm}$ in diameter when detected incidentally. However, symptoms occur when adrenal cysts become large enough to cause pain and gastrointestinal disturbances or when they become palpably enlarged. They may also be a consequence of intracystic bleeding or infection. Less frequent presentations include hypertension or spontaneous rupture of the cyst [12,14]. Management of giant adrenal cysts represents a challenge regarding the peri-operative diagnosis and the surgical procedure of resection or adrenalectomy. Indications for surgical intervention include a size exceeding $10 \mathrm{~cm}$ in diameter, the presence of symptoms, endocrine abnormalities, intracystic bleeding and suspicion of malignancy $[4,15]$. As early as 1908 , Doran urged complete excision of the cyst, although marsupialization and incision and drainage were frequently carried out by his contemporaries [1]. 
Without doubt, complete removal is the current treatment of choice, and laparoscopic adrenalectomy, where available, is the gold standard. Ultrasound-guided percutaneous drainage is a known safe alternative, especially when there is no doubt concerning the diagnosis [16]. Open complete surgical excision (adrenalectomy) is also a valid alternative which we offered to our patient based on the lack of a definitive preoperative diagnosis and the huge size of the cyst. The choice of access may be governed by the surgeon's preference. Clinically, the differential diagnosis of adrenal cysts is variable and includes any lesion that can present as an upper abdominal mass.

\section{Case Report}

A 35-year-old woman presented with a history of distension of the right flank and the upper quadrant of the abdomen. The patient was hospitalized for further examination and treatment of her symptoms. There was no history of trauma. Physical examination revealed a soft palpable mass in the right side of the abdomen. Her laboratory data on admission were within the normal range, including her hormone levels. Ultrasonography (US) showed that the mass was cystic with a homogeneous anechoic level. On contrast enhanced CT, a giant cystic mass measuring $20 \times 16 \times 25$ $\mathrm{cm}$ was identified beside the lateral segment of the liver, the lower pole of right kidney, and the ascending colon, which compressed the pancreas to the ventral side. we could not predict the origin of the cyst before the operation (Figure 1). The differential diagnoses considered included a renal cyst, pancreatic cystic lesion, retroperitoneal mucinous adenocarcinoma and adrenal cyst. As the patient desired relief from abdominal distension, we decided to perform surgical treatment for therapeutic diagnosis. We chose open laparotomy because of the risk of malignancy owing to the large size of the cyst.

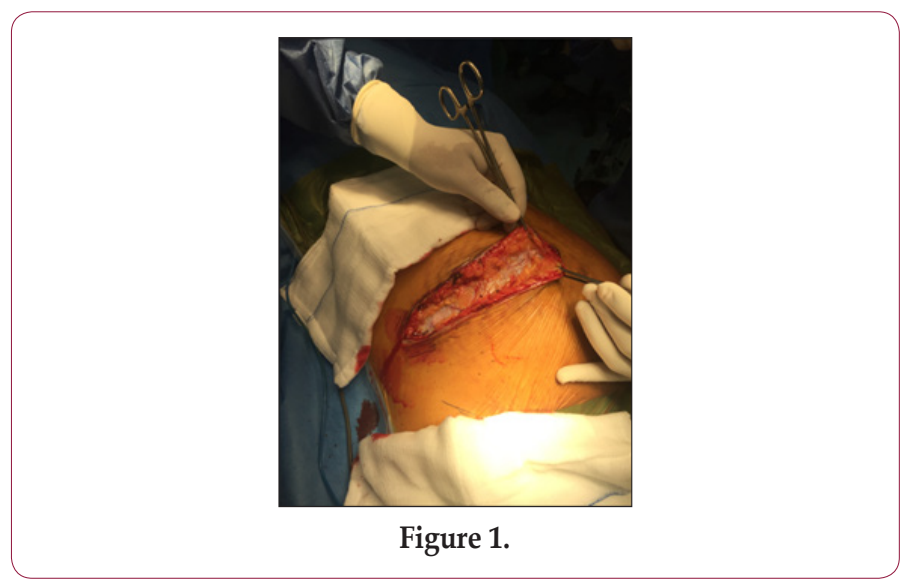

Under general anaesthesia, the patient was placed in the supine position. We made a right transverse subcostal abdominal incision. Intraoperative findings confirmed that the cystic mass neighbored the ascending colon, pancreas, and right kidney, but it also adhered to the right adrenal gland (Figures 2-7). We confirmed that the mass was derived from the right adrenal gland. Partial adrenalectomy was required for complete resection of the mass. The resected mass was about $21 \mathrm{~cm}$ in diameter, with over 2000 $\mathrm{mL}$ of brown-red serous fluid. No perioperative complications were encountered. The fluid of the cyst was found to contain no malignant component by cytological diagnosis. Hematoxylin-eosin staining indicated that the wall of the cyst consisted of a single flattened cell-lining. The cell lining was microscopically supposed to be derived from endothelium or mesothelium by hematoxylineosin staining (Figures 8-16). To confirm the origin of the single cell-lining, immunostaining for podoplanin (D2-40), alpha-inhibin, cytokeratin AE1/AE3 was performed. Immunohistochemically, the single cell lining of the cyst was strongly positive for D2-40 (Figures 4, 8-10), and negative for cytokeratin AE1/AE3 and alpha-inhibin, but the adrenal cortex or medulla was positive for alpha-inhibin. To confirm the origin of the lining further, additional immunostaining for calretinin and Wilms Tumor Suppressor Gene (WT1) was performed. As a result, the lining was negative for calretinin. These findings demonstrated that the wall of the cyst was not derived from the mesothelium.

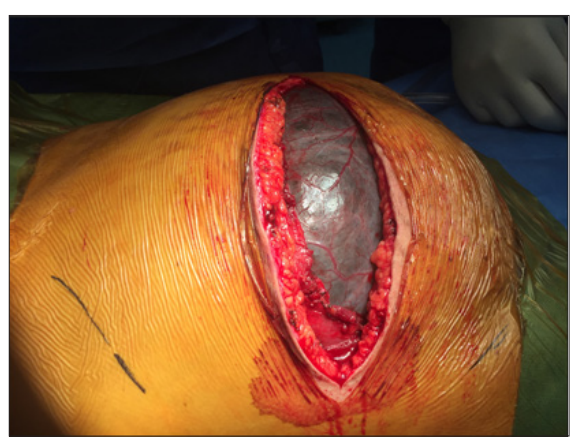

Figure 2.

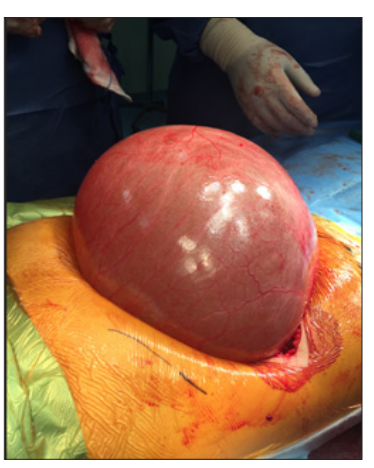

Figure 3.

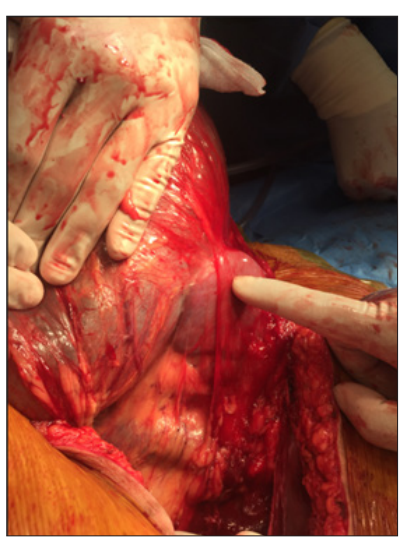

Figure 4 . 


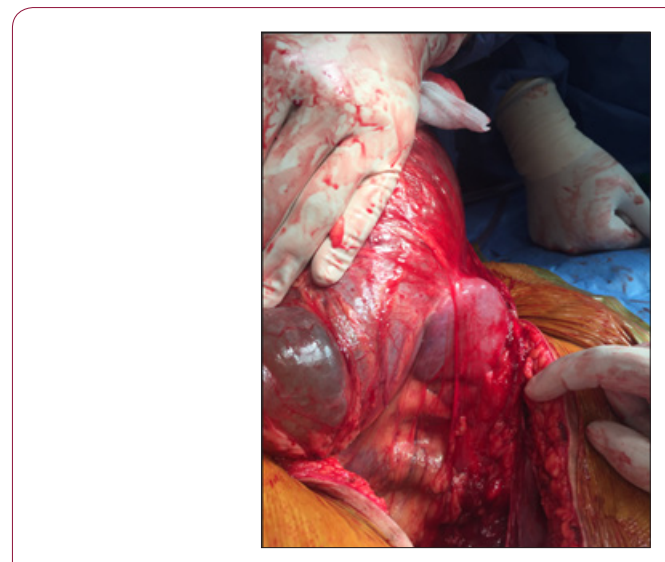

Figure 5.

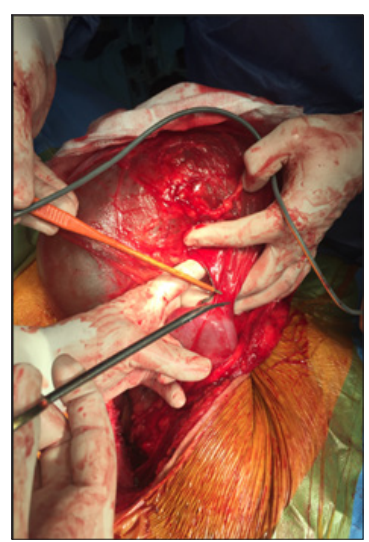

Figure 6.

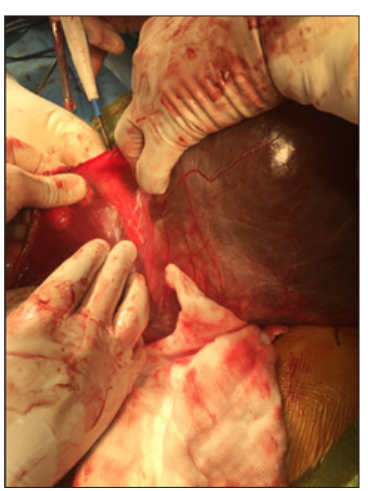

Figure 7.

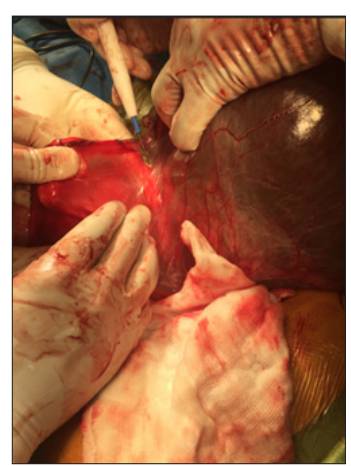

Figure 8 .

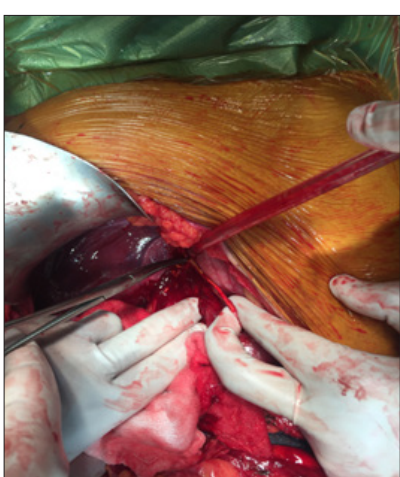

Figure 9.

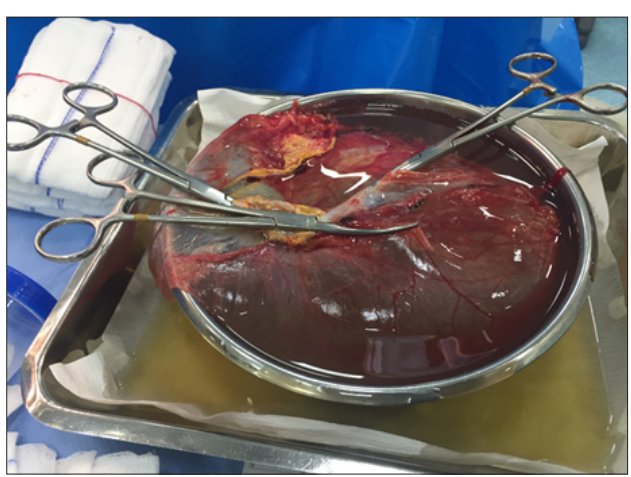

Figure 10.

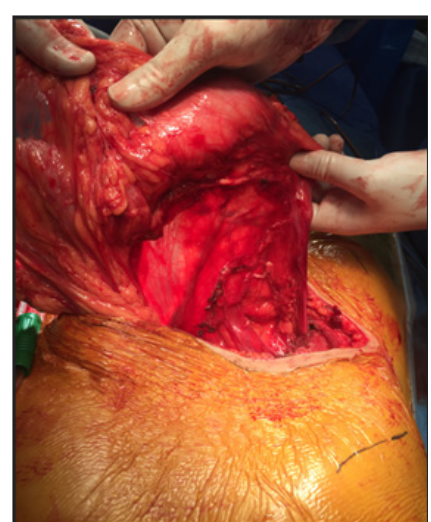

Figure 11.

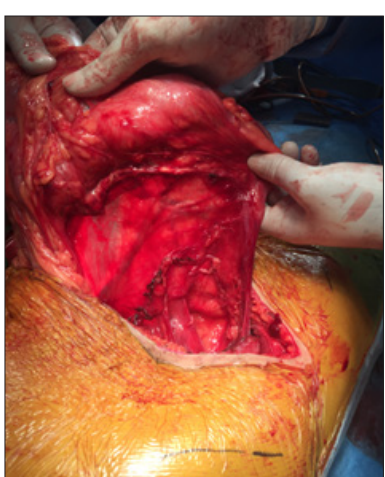

Figure 12. 

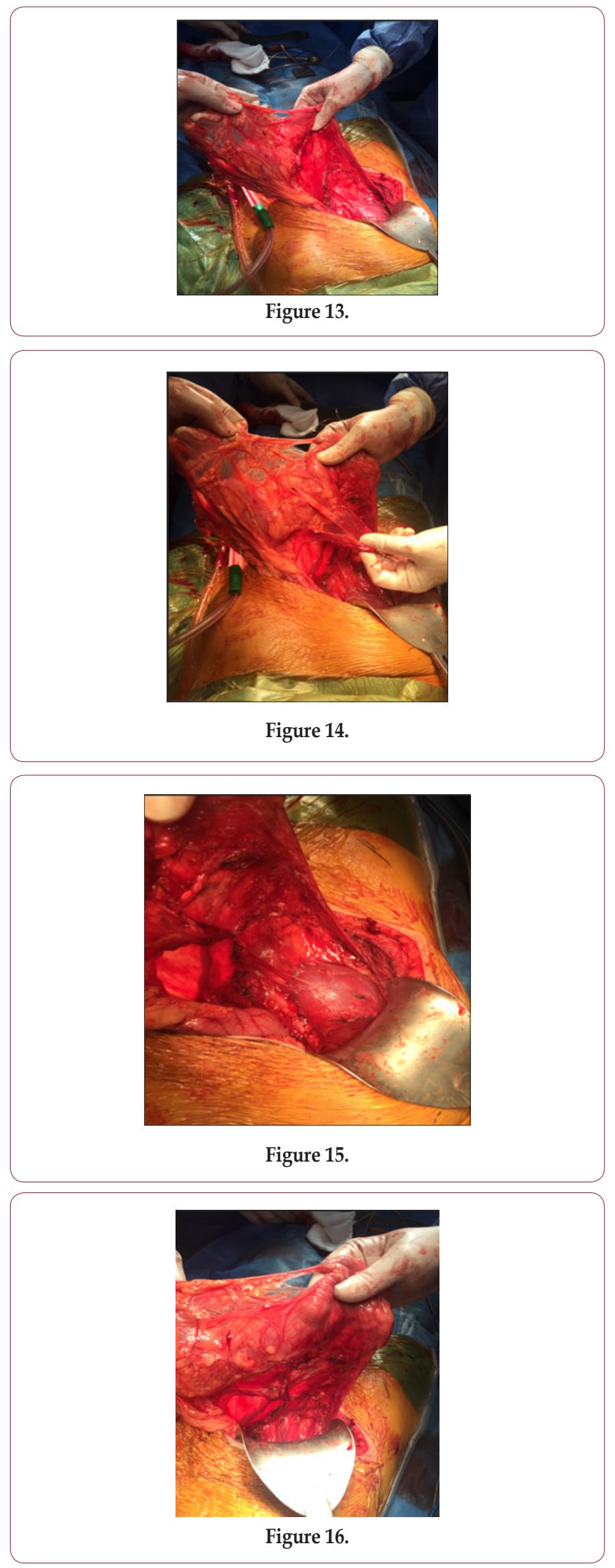

\section{Discussion}

Adrenal pseudo cysts are mainly benign and are uncommon entities. Adrenal pseudocysts may occur at any age but are often found in the fifth to sixth decade of life. Adrenal pseudo cysts constitute clinical entities with a malignancy potential of 7\%. 29 Adrenal cysts can develop within benign or malignant tumors, such as adenoma, hemangioma, pheochromocytoma, or malignant hemangioendothelioma Asbes house et al. 29 classified nonneoplastic adrenal pseudo cysts into 4 groups: endothelial (45\%), pseudo or hemorrhagic (39\%), epithelial (9\%), and parasitic (7\%). The symptoms associated with benign cysts are related to the position and size of the lesions. Pain, gastrointestinal disorders, dyspnea or a palpable mass can be observed in large cysts. Patients may present with acuteabdominal findings ifintracystichemorrhage or rupture occurs. Malignancy should be suspected in the presence of symptoms that are known to be caused by functioning neoplasms, such as Cushing syndrome, hyperadrenalism, hirsutism in women, acne and balding in men, and hypertension. An exact diagnosis is clinically important because an adrenal cyst more than $5 \mathrm{~cm}$ in diameter carries an increased risk of malignancy. For a radiological diagnostic work-up, CT and MRI are useful. Friedlich et al. have shown that benign and malignant adrenal masses can be identified using CT with a sensitivity of 85 to $100 \%$ and a specificity of 95 to $100 \%$. Magnetic resonance imaging is also a precise imaging modality for exploring adrenal masses and is important especially for masses undetected by CT.

Asbes house et al., had reported preponderance of about 3:1. Giant adrenal cysts are more likely to be left-sided (55.5\%), and all reported cases were of unilateral cysts (100\%). The greatest dimension of the cysts ranged from 20 to $50 \mathrm{~cm}$ (mean: $26.5 \mathrm{~cm}$ ). Most of the reported symptoms were pain, distention, and the findings of a palpable mass. Minor symptoms included gain or loss of weight, and gastrointestinal disturbances, such as nausea, or vomiting; however, the preoperative diagnosis was inaccurate or undetermined in the other cases. As giant adrenal cysts over 20 $\mathrm{cm}$ in diameter tend to adhere to many different adjacent organs, it can be difficult to detect the origin of the cysts. Asbes house et al. reported that malignancy of the adrenal lesions correlated with the size of the lesions. 29 No adrenal cyst without an associated adrenal neoplasm has been found to be a functioning lesion. 13,33 In contrast, there were no neoplastic and functioning cysts reported as giant adrenal cysts Therefore, we suggest that excessively large cysts and cysts with no function are less likely to be malignant. Of the previously reported giant adrenal cysts, 19 were pseudocysts (70.3\%); 4 were endothelial cysts (14.8\%); 1 was a lymphangioma (endothelial cyst, 3.7\%); 1 was an epithelial cyst (3.7\%); and 2 were of unspecified (7.4\%). Endothelial cysts are the most common type of adrenal cysts, which consist mostly of adrenal true cysts. Adrenal endothelial cysts are classified into angiomatous and lymphatic cysts. A lymphatic type of endothelial cyst is a more common type of adrenal cyst. An angiomatous type of endothelial cyst is the most uncommon form of adrenal cyst. 


\section{Conclusion}

Although there are no established guidelines for adrenal pseudo cysts, surgical intervention is generally indicated for adrenal cysts when they are hormonally functional, symptomatic, parasitic, malignant, enlarging or more than $5 \mathrm{~cm}$ in diameter. Percutaneous aspiration or drainage has been suggested as an alternative treatment; however, it should be limited to the conditions when the cyst is not hormonally active and malignancy can be ruled out. It can lead to temporary improvement of the symptoms, but it is a palliative treatment, whereas surgical excision provides for not only removal of the cyst wall, but also a definite pathological diagnosis (Figures 14-16) and can achieve cure. Adrenal pseudo cysts can be managed by an open or laparoscopic approach. Laparoscopic management has the advantage of less pain, rapid recovery, and better cosmesis. Parnaby et al. suggested that there is no significant difference in the outcome for all tumor sizes. However, aspiration of the content of the cyst was required to achieve successful laparoscopic adrenalectomy in some cases We suggest caution as aspiration of the content of the cyst can lead to tumor cell dissemination of a malignancy. In the present case, we did not choose laparoscopic management for the following reasons. First, because the cystic mass was over $20 \mathrm{~cm}$ in diameter, malignancy could not be eliminated. Second, aspiration of the cyst is needed to achieve a better intraoperative when using laparoscope; however, there would be a potential for tumor cell dissemination by aspiration of the cyst in case of malignancy. We suggest that laparoscopic surgery be used when intraoperative view can be obtained without aspiration of the cyst. Prior to cosmesis, maintaining oncological principles is the most important factor in choosing the operation method.

\section{References}

1. Carvounis E, Marinis A, Arkadopoulos N, Theodosopoulos T, Smyrniotis V (2006) Vascular adrenal cysts a brief review of the literature. Arch Pathol Lab Med 130(11): 1722-1724.

\section{ISSN: 2574-1241}

DOI: $10.26717 / B J S T R .2018 .10 .001890$

Frais Alaswad. Biomed J Sci \& Tech Res

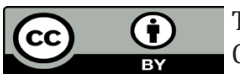

This work is licensed under Creative Commons Attribution 4.0 License

Submission Link: https://biomedres.us/submit-manuscript.php
2. Wieneke JA, Thompson LDR (2006) Non-neoplastic lesions of the adrenal gland. In: Thompson LDR, Endocrine pathology. In: Goldblum JR, Foundations in diagnostic pathology, Churchill Livingstone Elsevier, Philadelphia, PA, 5: 183-204.

3. Aloraifi F, O‘Brien G, Broe P (2008) Giant adrenal pseudocyst treated laparoscopically: Case report and review of the literature. Open Surg J 2: $39-42$.

4. Wang LJ, Wong YC, Chen CJ, Chu SH (2003) Imaging spectrum of adrenal pseudocysts on CT. Eur Radiol 13: 531-535.

5. Kim BS, Joo SH, Choi SI, Song JY (2005) Laparoscopic resection of an adrenal pseudocyst mimicking a retroperitoneal mucinous cystic neoplasm. World J Gastroenterol 15(6): 2923-2926.

6. Kar M, Pucci E, Brody F (2006) Laparoscopic resection of an adrenal pseudocyst. J Laparoendosc Adv Surg Tech A 16(5): 478-481.

7. Papaziogas B, Katsikas B, Psaralexis K, Makris J, Chatzimavroudis G, et al. (2006) Adrenal pseudocyst presenting as acute abdomen during pregnancy. Acta Chir Belg 106: 722-725.

8. Demir A, Tanidir Y, Kaya H, Turkeri LN (2006) A giant adrenal pseudocyst: Case report and review of the literature. Int Urol Nephrol 38(1): 167-169.

9. Sroujieh AS, Farah GR, Haddad MJ, Abu-Khalaf MM (1990) Adrenal cysts: Diagnosis and management. Br J Urol 65: 570-575.

10. Gaffey MJ, Mills SE, Fechner RE, Bertholf MF, Allen MS (1989) Vascular adrenal cysts: A clinicopathologic and immunohistochemical study of endothelial and hemorrhagic (pseudocystic) variants. Am J Surg Pathol 13: 740-747.

11. Erickson LA, Lloyd RV, Hartman R, Thompson G (2004) Cystic adrenal neoplasms. Cancer 101: 1537-1544.

12. Favorito LA, Lott FM, Cavalcante AG (2004) Traumatic rupture of adrenal pseudocyst leading to massive hemorrhage in retroperitoneum. Int Braz J Urol 30: 35-36.

13. Sioka E, Symeonidis D, Chatzinikolaou I, Koukoulis G, Pavlakis D, et al. (2011) A giant adrenal cyst difficult to diagnose except by surgery. Int J Surg Case Rep 2: 232-234.

14. Khilnani GC, Kumar A, Bammigatti C, Sharma R, Gupta SD (2008) Hemorrhagic pseudocyst of the adrenal gland causing acute abdominal pain. J Assoc Physicians India 56: 379-380.

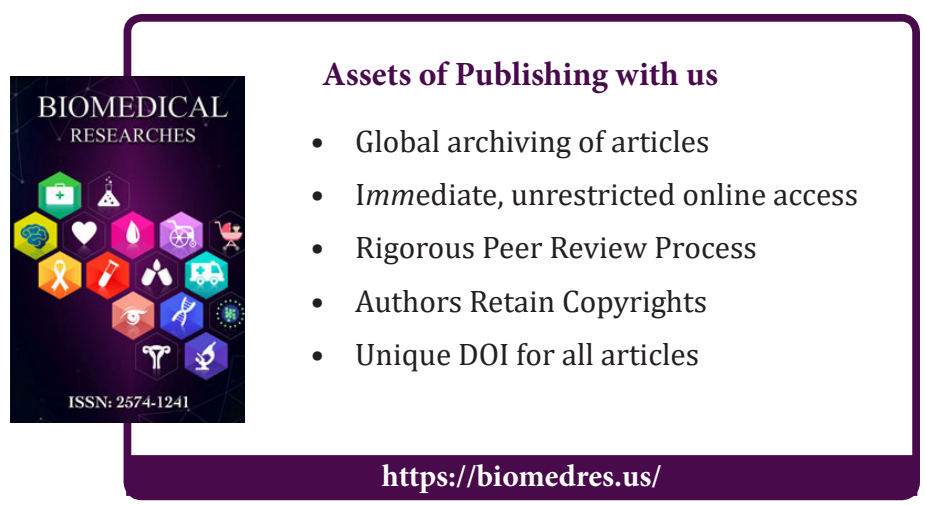

